SHS Web of Conferences 20, 01011 (2015)

DOI: $10.1051 /$ shsconf/20152001011

(C) Owned by the authors, published by EDP Sciences, 2015

\title{
Pour une analyse phénoménolinguistique de l'identité en interaction numérique
}

\section{Towards a phenomenolinguistic analysis of identity construction in digital interactions}

Samira Ibnelkaïd $\mathrm{d}^{1, a}$

${ }^{1}$ Laboratoire ICAR - Université Lumière Lyon 2

\begin{abstract}
Résumé. L'identité forme une notion complexe présentant des caractères instable et situationnel. Ce concept ne désigne pas une entité homogène et cohérente, mais renvoie aux interactions sociales (Greco \& Mondada, 2014 [1]). Et dans l'inconstance et l'inconsistance des nouveaux cadres et rapports sociaux induits part l'ère numérique, la quête identitaire se révèle permanente et les ressources multiples et complexes. Face à ces nouvelles « ressources sociotechniques » (Denouël, 2008 [2]), un nouveau cadre d'analyse nous semble nécessaire. Si le domaine de la linguistique concourt à l'étude de la construction langagière de l'identité en interaction, au-delà l'appui de la phénoménologie nous permet d'appréhender les dimensions phénoménale et intersubjective de l'engagement des sujets et de leurs identités.
\end{abstract}

\begin{abstract}
Identity forms a complex notion that should be understood not as a coherent and consistent entity but as a dynamic coconstruction between individuals especially during verbal interactions (Greco \& Mondada, 2014 [1]). Furthermore today's digital technologies offer new ways of communicating and expressing oneself (Denouël, 2008 [2]). And individual's search for identity is relentless. Therefore a new frame of identity analysis is required. If interactional analysis contributes to study the verbal construction of identity, phenomenology allows us to go beyond and apprehend the phenomenal and intersubjective dimensions of identity construction.
\end{abstract}

\section{Introduction}

L'identité forme une notion complexe présentant des caractères dynamique et interactionnelle. Ce concept ne peut en effet être perçu de manière essentialiste et objectiviste ; il ne désigne pas une entité homogène et cohérente, mais renvoie à la pratique des groupements humains ; "c'est dans leurs pratiques quotidiennes que les membres d'une société construisent tacitement leur appartenance à un groupe, leur caractère individualisant, leurs identités multiples et situées » (Greco \& Mondada, 2014 : 7 [1]). Aussi convient-il de privilégier l'interaction entre individus comme unité d'observation (Aymes \& Péquignot, 2000). Les interactions consistent en effet à produire des images de Soi et de l'interlocuteur ainsi qu'à les négocier. Et désormais, dans l'inconstance et l'inconsistance des nouveaux cadres et rapports sociaux induits part l'ère numérique, la construction identitaire se révèle permanente et les ressources multiples et complexes. L'identité se déploie en effet à l'écran par des ressources symboliques — tant linguistiques que corporelles et techniques — se trouvant à la disposition des interactants qui cherchent à se positionner face à l'altérité. Et l'hypermodernité ouvre un nouvel univers de communication qui augmente et modifie la capacité humaine de manipulation symbolique (Levy, 2013 : 16). L'usage de ces nouvelles « ressources sociotechniques » (Denouël, 2008 [2]) dans la construction des identités a fait émerger la notion d'identité virtuelle souvent perçue comme fictive, irréelle. Or le virtuel existe ; il existe en puissance (Lévy, 1995). Le virtuel se définit comme un « hors-là », en ce sens qu'il consiste à exister - du latin sistere, être placé, et du préfixe ex,

a Auteur de correspondance : samiraibnelkaid@gmail.com

This is an Open Access article distributed under the terms of the Creative Commons Attribution License 4.0, which permits unrestricted use distribution, and renroduction in anv medium provided the orioinal work is nronerlycited 
hors de - son essence est dans la sortie (Serres, 1994). L'identité virtuelle est un phénomène s'associant à l'expression de soi, il s'agit de prendre existence à l'écran pour se manifester à autrui.

Dès lors comment rendre compte de la construction de l'identité en interaction numérique ? La complexité que représente son analyse dans un corpus numérique nous semble imposer le recours à une démarche pluridisciplinaire. Une étude linguistique seule nous paraissant insuffisante dans l'appréhension du phénomène identitaire à l'ère numérique, nous proposons de l'associer à une approche phénoménologique. En effet, si le domaine de la linguistique concourt à l'étude de la construction langagière de l'identité en interaction, au-delà l'appui de la phénoménologie nous permet d'appréhender les dimensions phénoménale et intersubjective de la manifestation de soi et de la prise d'existence à l'écran. Notre proposition d'une analyse phénoménolinguistique de l'identité en interaction numérique, se justifie par l'idée selon laquelle « dès lors que l'Homme se sert du langage pour établir une relation vivante avec lui-même ou avec ses semblables, le langage n'est plus un instrument, n'est plus un moyen, il est une manifestation, une révélation de l'être intime et du lien psychique qui nous unit au monde et à nos semblables » (Merleau-Ponty, $1945: 229$ [3]). Une telle approche nous invite alors à consentir que «si nous réussissons à comprendre le sujet, ce ne sera pas dans sa pure forme, mais en le cherchant à l'intersection de ses dimensions » (Ibid. : 470). Aussi proposons-nous pour cadre d'analyse, une démarche pluridisciplinaire visant l'appréhension de la complexité du phénomène intersubjectif de co-construction des identités en interaction numérique.

Nous nous attacherons ici à expliciter cette démarche et l'illustrer sur un corpus d'interactions numériques entre participants géographiquement distants et se rencontrant pour la première fois par écran. Cette rencontre se réalise au moyen de trois modes interactionnels numériques successifs : asynchrone écrit (forum), quasi-synchrone écrit (tchat), synchrone audiovisuelle (visio). Pour les besoins de cet article nous porterons notre attention sur le premier mode. Notre approche phénoménolinguistique des interactions numériques se fonde sur l'analyse du discours en interaction (Kerbrat-Orecchioni, 2005 [4]) et la phénoménologie de la perception (Merleau-Ponty, 1945 [3]).

Nous proposons ici dans un premier temps de dresser une épistémologie de la notion d'identité. Dans un deuxième temps nous mettrons en lumière les aspects linguistiques et phénoménologiques de la construction identitaire en interactions présentielle et numérique. Nous pourrons alors mettre en application notre approche phénoménolinguistique sur notre corpus d'interaction numérique et faire part de nos résultats.

\section{L'identité comme construction intersubjective}

Étudier la construction de l'identité individuelle suppose de prendre en considération les caractères instable et situationnel d'un tel concept. Une tentative d'épistémologie de son contexte d'apparition révèle rapidement la jeunesse de cette notion.

\subsection{L’identité : un concept répandu mais récent}

Si notre époque hypermoderne exalte l'identité et en promeut la construction, l'épistémè pré-moderne l'ignore. La pré-modernité se caractérise en effet notamment par l'absence d'individualité. La configuration de la société fondée sur une hiérarchie de statuts et rangs hérités impliquait l'absence d'interrogation de l'individu sur son sort ; « l'individu n'a pas à se poser la question de ce qu'il va faire ou de ce qu'il va être et devenir car le chemin est déjà tracé » (Dieu \& Dubois, $2012: 2$ ). L'époque moderne sonne alors le glas de la hiérarchie verticale et de l'autorité arbitraire et instruit un déplacement de l'autorité divine ou ancestrale à l'autorité de l'Homme par l'Homme. C'est le scepticisme propulsé par les grandes découvertes, les Lumières, les réformes et révolutions qui initiera la remise en cause des certitudes passées. Le sujet se trouve alors responsabilisé et impliqué dans sa propre trajectoire; c'est l'individuation, la subjectivité — l'expérience que le sujet pensant fait de luimême. Pour autant, l'époque moderne rend compte d'une identité unique, figée, déterminée par la raison. Cette solidité de l'identité se trouvera fragilisée par l'ère postmoderne au cours de laquelle l'individu se fragmente, les identités se démultiplient. L'individu est «plus autonome mais il n'est pas 
seul » (Ibid.). La construction du Soi est irrémédiablement attachée à l'Autre. Les identités résultent de l'interaction entre les subjectivités et leur prise en compte mutuelle- l'intersubjectivité. L'horizontalité des relations interindividuelles est liée à la quête de repères de la part des sujets qui se comparent alors à autrui afin de trouver reconnaissance et confirmer leur identité. Dans l'échange intersubjectif et le partage dialogique de son expérience, l'individu recherche la validation par l'Autre de ses propres modes de croire, en fonction desquels il agit (Taylor, 1992). Mais la rapidité et l'intensité des flux de communication et de relation de l'hypermodernité introduisent une surenchère dans la construction du Soi et de l'Altérité. Les sociétés hypermodernes « sont emportées par l'escalade du toujours plus, toujours plus vite, toujours plus extrême dans toutes les sphères de la vie sociale et individuelle » (Lipovetsky, 2010).

\subsection{Identité et altérité}

L'identité s'accomplit donc désormais dans l'interaction sociale et cet accomplissement se réalise sous le regard d'autrui par une variété de ressources langagières, corporelles et techniques se trouvant à la disposition des interactants qui cherchent à se positionner face à l'altérité. Ainsi la communication interindividuelle ne relève pas du simple transfert d'informations. Elle contient, de fait, bien plus, dans la mesure où nous communiquons aussi pour « nouer des relations, partager des émotions et des sentiments, agir sur autrui, séduire ou attaquer, conforter notre identité ou celle des autres » (Lipiansky, 1993 : 31 [5]). Les interactions sociales consistent en effet également à produire des images de Soi et de l'interlocuteur ainsi qu'à les négocier. C'est par et dans la communication que les êtres se construisent et se définissent ; " ils se découvrent alors pluriels selon les situations communicationnelles » (Ibid.). Il s'agit alors pour le chercheur en Sciences du Langage " d'appréhender les identités dans leur incessante mobilité et fluctuation et de concevoir leur stabilité comme le fruit d'un travail constant et minutieux " (Greco \& Mondada, 2014: 20 [1]). Nous favorisons donc une conceptualisation dynamique et dialectique de l'identité qui s'apparente plus à un processus identitaire qu'à une entité stable et permanente (Gaulejac, 2002 : 175). Cette conceptualisation dialectique de l'identité renvoie au fait que le terme même est porteur d'une contradiction fondamentale : la similitude et la différence. Tout individu s'identifie tant par ce qu'il a de semblable à l'autre que par ce qui le distingue de cet autre.

\section{Une approche phénoménolinguistique de la construction identitaire en interaction}

Les identités ne peuvent être perçues «comme un «extra-linguistique » se situant en dehors du langage mais comme étant imbriquées dans la séquentialité de l'action ainsi qu'avec une multitude de ressources sémiotiques » (Greco \& Mondada, $2014: 20$ [1]). L'identité relève ainsi de la construction langagière et multimodale mais également du phénomène en ce qu'elle consiste à prendre existence aux yeux d'autrui. Notre choix de mettre en regard le domaine de l'analyse des interactions et celui de la phénoménologie repose également sur le lien épistémologique entre ces deux disciplines - la première étant l'héritière de la seconde. En effet, l'analyse interactionnelle de Sacks, Schegloff et Jefferson trouve ses origines dans l'ethnométhodologie de Garfinkel, et ce-dernier a été inspiré par la sociologie phénoménologique de Schütz et la phénoménologie de Husserl et Merleau-Ponty (Corcuff, 1981 ; Gullich, 1991 ; Angermüller, 2010 ; Berger \& Luckmann, 2012 ; ...). Analyse des interactions et phénoménologie sont donc intrinsèquement liées et nous entendons les faire communiquer ici afin d'appréhender la complexité de l'identité comme phénomène interactionnel.

\subsection{L'identité en interaction...}

Les rencontres forment le lieu de présentation de soi à l'autre. Goffman explique que « quand une personne se présente aux autres, elle projette, en partie sciemment et en partie involontairement, une 
définition de la situation dont l'idée qu'elle se fait d'elle-même constitue un élément important » (Goffman, 1973 : 229 [6]). Lorsqu'un individu est mis en présence d'autres personnes, celles-ci mobilisent ou cherchent des informations à son sujet dans le but de contribuer à définir la situation de communication, prévoir ce que chacun en attend, savoir comment interagir. Si les participants ne connaissent pas l'individu, ils peuvent effectuer des postulats à partir de son apparence et sa conduite, des expériences passées, ce que l'individu dit de lui-même. L'acteur donne une expression de luimême de laquelle les autres doivent retirer une impression (Goffman, 1973 : 11-12 [6]). Lipiansky parle d'《 identité situationnelle » (Lipiansky, 1993 : 32 [5]). Celle-ci se co-construit, se reproduit, se confirme ou au contraire est remise en cause au cours des interactions entre soi et autrui. Ce « travail identitaire " (Mondada \& Greco, 2014 [1]) se réalise notamment au moyen des "identèmes » (Neyraut, 2008 [7]) émis au cours de l'interaction. L'identème désigne «la plus petite unité significative d'une identification dont elle représente à la fois le substrat et la fin » (Neyraut, 2008 : 173 [7]). Les identèmes véhiculent l'identité des interactants et forment le résultat temporaire du travail identitaire au cours de l'interaction. Nous verrons au cours de notre analyse que l'identème peut prendre plusieurs formes - linguistique, corporelle, technique - et passer par plusieurs états - du virtuel au réel.

\section{2 ... numérique}

Selon Goffman, la vie sociale est une scène pour autant que les membres d'une société accomplissent leurs activités ordinaires dans le champ d'une perception mutuelle ou dans la présence immédiate d'autrui de telle sorte qu'ils sont physiquement en présence de la réponse de l'un et de l'autre (Quéré, 1989 : 54). Les expressions corporelles ainsi produites constituent des symboles signifiants servant de base d'inférence dans le raisonnement pratique et assure une fonction spécifique dans l'organisation des rencontre (Ibid. : 55). Or il est des rencontres qui ne se déroulent pas en coprésence corporelle des interactants. Si dans le réel, le corps donne d'emblée existence à la personne, lui permettant de se manifester aux yeux des autres et ainsi de construire son identité par différenciation, à l'écran, il est nécessaire que la personne prenne existence car si elle n'agit pas, elle est invisible pour l'autre (Georges, $2008: 2$ ). C'est alors la saisie d'informations qui l'inscrit dans l'interface numérique et conditionne sa construction identitaire. Il est question d'identité en interaction numérique, celle-ci pouvant être définie comme " une transposition graphique, sonore et visuelle d'une représentation en pensée façonnée par le Sujet dans le matériau de l'interface » (Georges, 2009 : 169 [8]). L'identité virtuelle ne renvoie donc pas à une identité fictive mais elle est au contraire censée refléter une identité réelle. L'individu construit une représentation abstractive de lui-même que Peirce nomme un « schéma- silhouette » de soi $(1978: 120)$. Georges reprend ce concept pour faire référence au fait que l'individu fait abstraction d'un certain nombre d'informations qu'il juge non pertinentes, et en choisit d'autres qui lui semblent plus adéquates, i.e. plus conformes à la représentation qu'il a de lui-même (2009 : 169 [8]) et de la situation d'interaction. La représentation de soi à l'écran se réalise par de nouvelles formes d'apparition corporelle. En effet les interactions numériques révèlent des traces corporelles monodimensionnelles (pseudonymes, émoticônes,...), bidimensionnelles (photos, avatars $2 \mathrm{D}$, profils,...) et tridimensionnelles (interactions vidéos, personnages virtuels,...) qui sont autant de repères cognitifs permettant de dessiner les caractéristiques physiques, les sensibilités et le comportement des interlocuteurs en ligne (Casilli, 2012 [9]).

\subsection{L’identité comme phénomène...}

L’origine étymologique du terme «phénomène » se trouve dans le verbe grec $\varphi \propto l v \varepsilon \sigma \theta \alpha l$ : apparaître, se montrer. Or comme apparaître est un mode privilégié de la rencontre ; il préfigure l'entrée en contact du sujet et de l'objet ou de l'autre, de leur décisive " prise de connaissance » (Heidegger, 1972 [10]). L'expérience d'autrui se concrétise dans le phénomène de la rencontre. C'est pourquoi les phénoménologues considèrent la rencontre comme un moment " où se joue et se rejoue, de façon largement pré-réfléchie, notre contact avec notre monde, nos prochains et nous-mêmes » (Vannotti \& 
Gennart, $2014: 1)$. L'intérêt est alors porté sur «l'existence comme mode d'être fondamentalement intentionnel ou ex-tatique (ex-sistere signifiant littéralement : se tenir hors) » (Ibid.). Ainsi, le monde phénoménologique ne consiste pas en un existant pur mais en un sens qui se construit et apparaît à l'intersection de mes expériences ainsi qu'à l'intersection de mes expériences et de celles de l'Autre, soit entre subjectivité et intersubjectivité. Ces dernières s'unissent « par la reprise de mes expériences passées dans mes expériences présentes, de l'expérience d'autrui dans la mienne » (Merleau-Ponty, 1945 : 15 [3]). Il existe ainsi un lien irréductible entre les sujets dans leur construction réciproque. C'est pourquoi, Sartre conclue qu' " autrui apparaît nécessaire à la constitution même de ce moi » (1943 : 278 [11]). La construction identitaire en interaction apparaît donc phénoménologique par essence.

\section{4 ...numérique}

Les études phénoménologiques ont jusqu'alors centré leur intérêt sur la relation entre soi et l'Autre et entre soi et l'objet délaissant de ce fait la médiation technique des relations interindividuelles. Or comme le souligne Vial " tous les phénomènes sont phénoménotechniques. Il y a une technicité fondamentale de l'apparaître » (Vial, 2013 : 19 [12]). En effet, pour qu'un Autre ou un objet apparaisse à un Soi et inversement, une médiation technique est nécessaire quelle qu'en soit la forme. La phénoménotechnique générale impulsée par Bachelard nous rappelle que l'" ontophanie » nécessite une technique tant pour se réaliser que pour être observée. Ce terme d'ontophanie est créé en

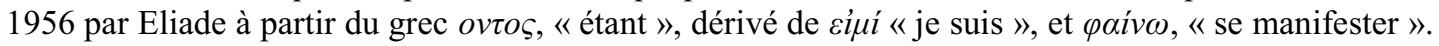
L'ontophanie fait ainsi référence à la manifestation, l'apparition, la révélation de l'être, de son existence ou de son essence (Eliade, 1956 : 134). Et les technologies numériques permettent désormais de se manifester à autrui à distance. Le virtuel a pris part au réel de sorte que les individus ont acquis de nouvelles habitudes perceptives et ont intégré les interactions en ligne dans leur quotidien. Ce que les technologies présentent à l'usager apparaît alors comme réel. Ce nouvel environnement techno-perceptif hybride se dénomme " l'ontophanie numérique » (Vial, $2014: 44$ [12]).

\section{Analyse phénoménolinguistique d'un travail identitaire}

La construction de l'identité en interaction numérique est donc un processus complexe à appréhender pour le chercheur en Sciences Humaines. Aussi cherchons-nous à participer à son appréhension à partir de notre approche pluridisciplinaire. Nous proposons ici d'illustrer notre démarche phénoménolinguistique par l'analyse d'une rencontre entre participants géographiquement distants échangeant pour la première fois, en ligne. Il s'agit d'étudiants de didactique des langues à Lyon (France) et à Berkeley (États-Unis). Les participants ont interagi une première fois par messages sur forum dédié consistant en de courtes présentations de soi. La semaine suivante, ils ont échangé par tchat durant une séance d'une heure avec les participants de leur choix. Et une semaine plus tard, ils ont commencé à interagir par appel vidéo Skype durant une heure une fois par semaine pendant six semaines. L'objet principal des discussions est la didactique des langues mais d'autres thématiques relevant de la rencontre interpersonnelle (vie du quotidien, loisirs, expériences, etc.) sont abordées au cours des échanges. Ne pouvant présenter ici l'analyse de l'ensemble du corpus, nous illustrerons notre approche en nous intéressant à un seul trinôme (en l'occurrence celui pour lequel nous disposons du plus de données enregistrées) et nous ferons un focus sur le tout premier échange (asynchrone écrit). 


\subsection{Présentation de soi en interaction écrite asynchrone}

Sur un Forum dédié au cours de didactique, les participants ont posté une présentation d'eux-mêmes.

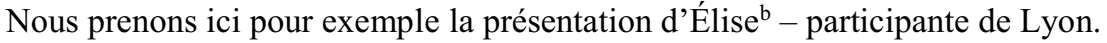

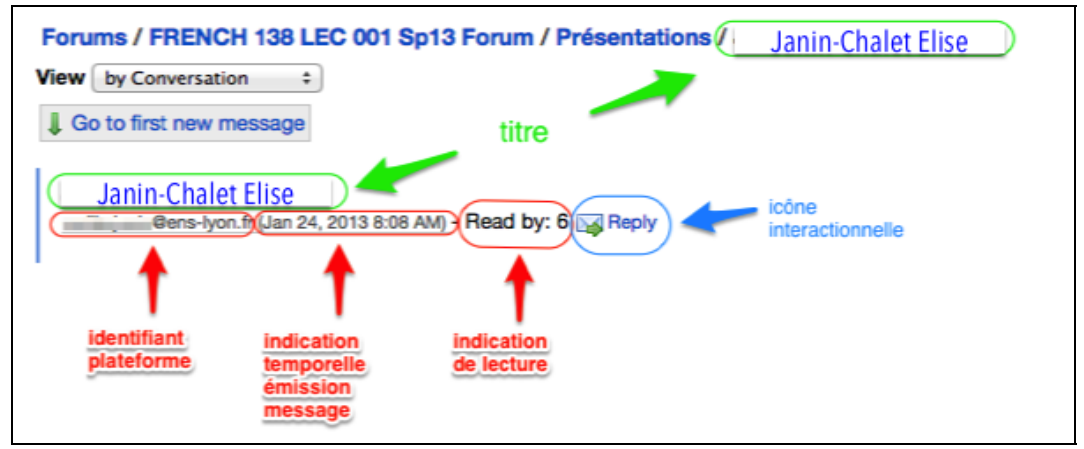

Figure1. En-tête présentation d'Élise.

Le travail identitaire apparaît ici co-construit par l'outil et le locuteur. En effet les identèmes sont émis d'une part par Élise elle-même qui choisit pour titre de message son nom de famille et son prénom, d'autre part par l'outil, via l'identifiant plateforme (l'adresse mail de la locutrice). À ces identèmes nominaux, s'ajoutent des identèmes d'activité (indication temporelle d'émission du message, indication de "popularité » du message - nombre de lecteurs) générés par l'outil. L'identification du locuteur respecte donc un schème numérique, identique pour tous.

Sous cet en-tête apparaît le message de présentation rédigé par Élise.

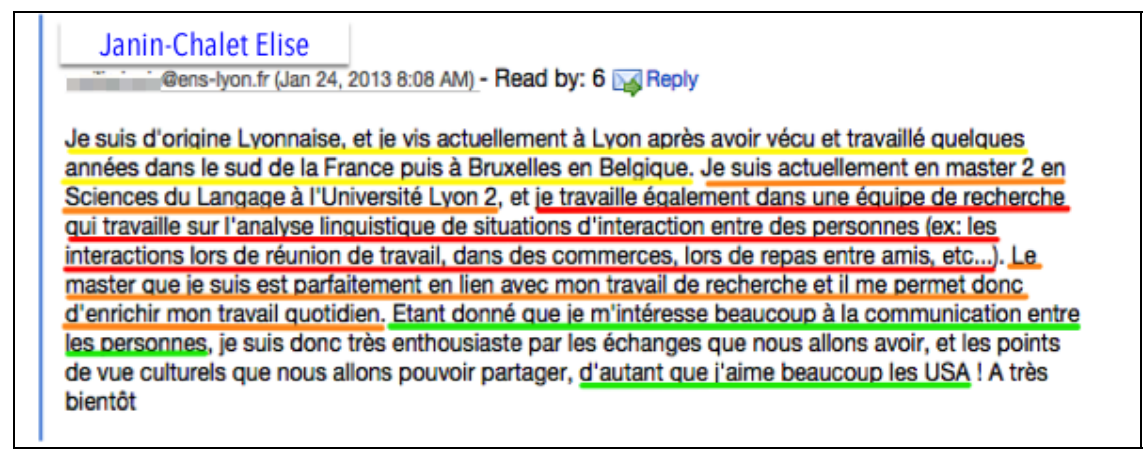

Figure 2. Texte de présentation d'Élise (nous soulignons).

Les identèmes sélectionnés et transmis par Élise dans sa présentation ne le sont pas de manière aléatoire. Ils font l'objet d'un découpage en ce que l'on nommera «champs identémiques ». Ces champs renvoient à son parcours géographique (en jaune dans l'image), sa formation (en orange dans l'image), sa profession (en rouge dans l'image) et ses centres d'intérêts (en vert dans l'image). La locutrice fait un travail identitaire de choix des champs identémiques jugés pertinents dans cette situation d'interaction et des identèmes appropriés au sein de ces champs. Elle ordonne par ailleurs ces champs identémiques en débutant sa présentation par son origine et en l'achevant par ses centres d'intérêts.

À ce stade de la rencontre, le travail identitaire est auto-initié et co-construit par le locuteur et l'outil. L'autrui-destinataire reste relativement inconnu - Élise ne sait pas précisément qui lira sa présentation. La sélection d'identèmes pertinents apparaît donc complexe car fondée uniquement sur des assomptions sur la situation d'interaction à venir. Les identèmes proposés tiennent du virtuel dans

\footnotetext{
b Par souci d'anonymat, nous remplaçons les noms par des pseudonymes.
} 
la mesure où ils ont pour fonction de sortir de l'anonymat, de manifester le locuteur, de prendre existence à l'écran. L'ontophanie n'est pas encore effective dans la mesure où l'apparition d'Élise n'est pas encore perçue par autrui. Il s'agit d'un stade pré-ontophanique que nous nommerons protophanie. Cette protophanie numérique se délimite à la manifestation du sujet à l'écran avant qu'autrui n'en prenne connaissance.

Au message de présentation d'Élise, Judy - une participante de Berkeley - répondra le message suivant.

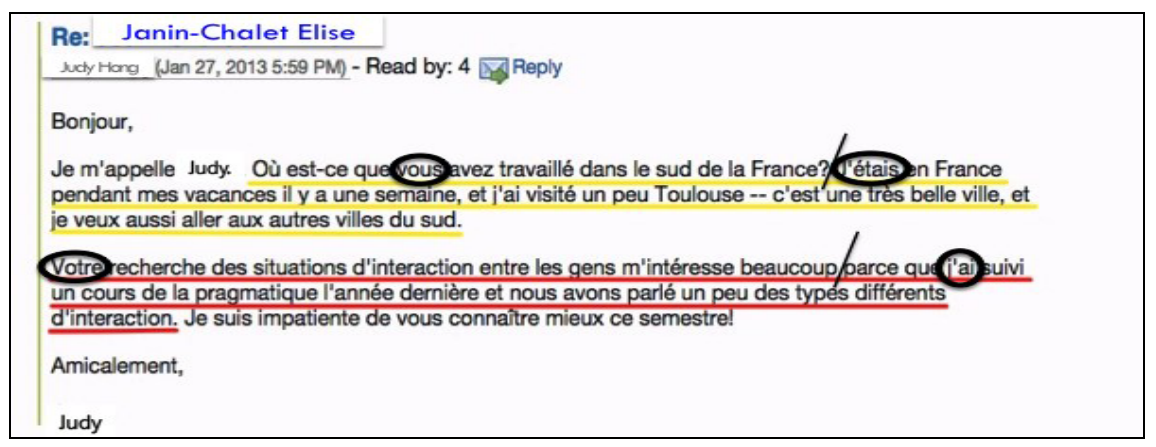

Figure 3. Réponse de Judy à la présentation d'Élise (nous soulignons et entourons).

L'intervention réactive de Judy au message d'Élise se réalise en asynchronie (message d'Élise envoyé le 24 Janvier et réponse de Judy postée le 27 Janvier). Aussi le message est-il constitué autant de questions portant sur l'identité d'Élise que d'informations portant sur l'identité de Judy. En effet, à chaque interrogation émise par Judy correspond une réponse la concernant. Au sein des quatre champs identémiques abordés par Élise dans sa présentation, deux ont retenu l'attention de Judy - son parcours géographique et sa profession. Et au sein du premier champ, Judy opère une nouvelle sélection, celle du séjour dans le sud de la France. Judy interroge alors Élise sur la ville exacte de son séjour pour le travail et associe cette interrogation à un identème propre considéré comme similaire et pertinent - Judy a visité Toulouse. Il en va de même concernant le second champ identémique - la recherche en interaction - que Judy reprend, à l'affirmative cette fois, pour l'associer de nouveau à un identème similaire - Judy a suivi un cours de pragmatique. Ainsi Judy effectue une tentative d'accordage identémique en proposant des identèmes propres similaires à ceux d'Élise. Cette dernière répondra à Judy par le message suivant.

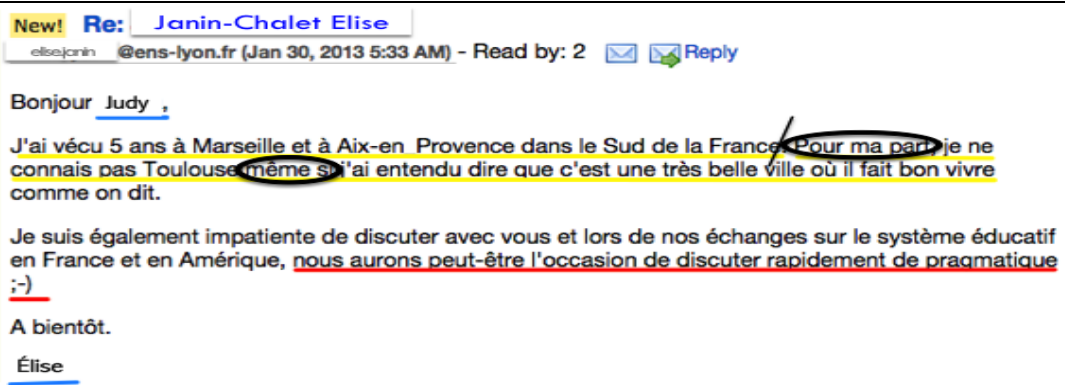

Figure 4. Réponse d'Élise à la réponse de Judy (nous soulignons et entourons).

En premier lieu, dans sa réponse, Élise ratifie l'identité nominale de Judy en l'adressant par son prénom dans sa salutation d'ouverture. Suite à cette ouverture, Élise d'une part répond à l'interrogation de Judy et d'autre part évalue la tentative d'accordage identémique. En l'occurrence cette tentative de Judy échoue dans la mesure où toutes deux n'ont pas visité la même ville. Cette évaluation négative pouvant être perçue comme un acte menaçant pour la face (Brown \& Levinson, 1978) de Judy, Élise l'associe à un adoucisseur introduit par «même si » et suivi d'un mélioratif 
«c'est une très belle ville ». Élise relève également la tentative d'accordage identémique lié à la pragmatique et l'évalue positivement en proposant d'en discuter ultérieurement.

Ainsi une structure interactionnelle émerge de ce travail identitaire en asynchronie écrite. Un premier tour de parole asynchrone ou «a-tour» (Mondada, 1999) émis par le locuteur 1 transmet une information identitaire $\mathrm{A}$ auquel un second tour de parole émis par le locuteur 2 transmet une information identitaire B similaire à A. Néanmoins l'interaction se déroulant en asynchronie, le travail identitaire fait l'objet d'un échange inséré avec un a-tour de L2 consistant en une demande précision sur l'information identitaire $\mathrm{A}$ et une réponse de $\mathrm{L} 1$ à cette demande de précision. Suite à ces deux échanges enchâssés, L1 évaluera positivement ou négativement la tentative d'accordage identémique. L'asynchronie augmente le risque d'évaluation négative dans la mesure où le monitoring progressif n'est pas possible.

1. L1: $a-F p p^{c}$-> Information identitaire $A$

2. L2 : a-Fie -> Demande de précision sur l'information identitaire $A$

3. L2 : a-Spp -> Information identitaire $B$ similaire à $A$

4. L1: $a$-Sie -> Information identitaire $A$ ' (précision sur $A$ )

5. L1: a-Tp -> Évaluation de l'accordage identitaire $A-B$

Cette structure interactionnelle de travail identitaire se retrouve dans l'ensemble des interactions asynchrones sur forum de notre corpus.

À ce stade de la rencontre, le travail identitaire est hétéro-initié et co-construit par les deux locuteurs via l'outil. L'autrui-destinataire se dévoile - en proposant ses identèmes propres. Les identèmes font l'objet d'une tentative d'accordage par l'interlocuteur qui a préalablement choisi le locuteur initial auquel répondre. L'interlocuteur actualise, par sa simple réponse, les identèmes du locuteur et en ratifie une partie - ceux qu'ils jugent intersubjectivement pertinents. Les identités se réélisent. Ce stade est celui de l'ontophanie : le sujet est perçu par autrui dans l'environnement numérique. Cette perception est technologiquement indicée par l'icône de lecture ("read by x ») et textuellement ratifiée par l'interlocuteur qui répond au locuteur initial.

$\mathrm{Au}$ sein de l'ensemble des identèmes potentiels que le locuteur initial pouvait communiquer à son interlocuteur en ligne, seule une partie a effectivement été exprimée. Ces identèmes exprimés par le locuteur sont alors reçus par l'interlocuteur qui effectuera une nouvelle sous-sélection des identèmes qui l'interpellent et sera demandeur de nouveaux identèmes.

Le travail identitaire tient autant du phénomène de révélation de soi à autrui et de confirmation intersubjective de cette révélation que du processus d'accordage identitaire mutuel.

\section{Résultats}

L'analyse des présentations écrites initiatives a révélé des spécificités dans la construction de la définition de soi à autrui. Ces présentations tiennent en effet de la sélection des identèmes perçus comme pertinents au sein d'un ensemble d'identèmes potentiels. Les locuteurs ne peuvent exposer leur « biographie » (Goffman, 1975) complète sur le Forum, ils doivent opérer des choix. Nous avons constaté que ces choix relèvent en premier lieu de champs identémiques (parcours géographique, professionnel, centre d'intérêts, etc.). De nouveau l'ensemble de ces champs n'est pas entièrement développé dans la présentation de soi. Une nouvelle sélection se produit en effet en second lieu. Les locuteurs déterminent les éléments à communiquer au sein de ces topics. Cette attribution identitaire auto-initiée est numériquement transmise et en attente de réception par les interlocuteurs. La réception par autrui fait alors suite à la sélection et à l'expression par soi. Mais la réception des informations identitaires par autrui ne suffit à confirmer l'élément identitaire exprimé. C'est la ratification de la part de l'interlocuteur qui réduit la distance entre identité pour soi et identité pour autrui. Cette ratification

\footnotetext{
c a-FPP : a-tour First Pair Part (première partie de l'échange asynchrone); a-SPP : a-tour Second Pair Part (seconde partie de l'échange asynchrone); a-Fie : a-tour First insert expansion (première partie de l'échange inséré); a-Sie : a-tour Second insert expansion (seconde partie de l'échange inséré); a-Tp : a-tour Third Part (troisième partie de l'échange asynchrone).
} 
peut prendre la forme d'une hétéro-reprise ou d'un énoncé interrogatif. Ce dernier a la double fonction de ratifier l'information identitaire sur lequel il porte et de solliciter d'autres éléments identitaires liés. Il apparaît que les identèmes passent par quatre états. Ces quatre états observés - sélectionné, exprimé, reçu, ratifié - nous semblent renvoyer au quadrivium ontologique développé par Lévy (1998 [13]). Ce dernier distingue potentiel, réel, virtuel et actuel. Les deux premiers tiennent de la substance et les deux derniers de l'événement. Les deux substances, potentiel et réel, se distinguent en ce que l'une est latente (potentiel), l'autre est manifeste (réel). Il en va de même pour les deux événements dont l'un est latent (virtuel) et l'autre est manifeste (réel).

\begin{tabular}{|l|c|c|}
\hline & Latent & Manifeste \\
\hline Substance & $\begin{array}{c}\text { Potentiel } \\
\text { (insiste) }\end{array}$ & $\begin{array}{c}\text { Réel } \\
\text { (subsiste) }\end{array}$ \\
\hline Évènement & $\begin{array}{c}\text { Virtuel } \\
\text { (existe) }\end{array}$ & $\begin{array}{c}\text { Actuel } \\
\text { (arrive) }\end{array}$ \\
\hline
\end{tabular}

Figure 5. Le quadrivium ontologique (Lévy, 1998 [13])

La substance latente qui insiste - le potentiel - renvoie dans notre analyse à l'identème se trouvant dans la biographie de l'individu, en attente de sélection. L'événement latent - le virtuel - est l'expression de cette information qui va la faire exister. L'identité virtuelle existe en puissance. Le virtuel se définit comme un " hors-là », en ce sens qu'il consiste à exister - du latin sistere, être placé, et du préfixe ex, hors de - son essence est dans la sortie (Serres, 1994 [14]). Cette énonciation identitaire arrive, elle s'actualise quand l'événement de son expression devient manifeste à autrui. Enfin, l'identité devient réelle dès lors qu'elle subsiste, qu'elle est une substance manifeste, qu'elle est ratifiée par autrui et reprise dans l'interaction.

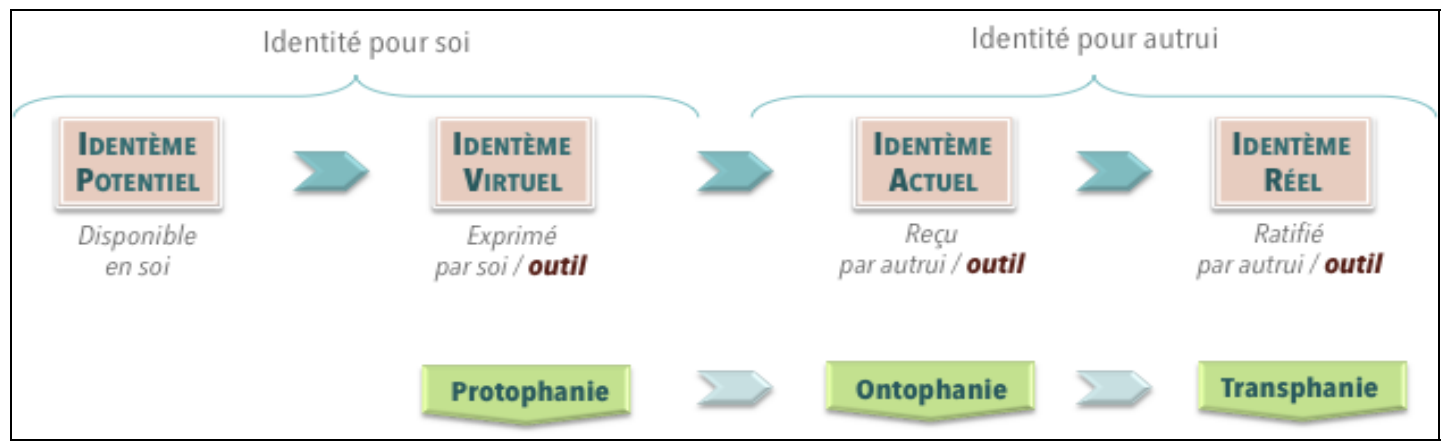

Figure 6. Les quatre états de l'identème ; le travail identitaire en interaction numérique

En outre, il apparaît que l'ontophanie ne constitue pas la seule forme d'apparition de la rencontre mais en forme la pierre angulaire. En se connectant sur la plateforme numérique (forum, tchat ou vidéo), les sujets apparaissent à l'écran mais cette apparition n'est perçue par autrui que lorsque luimême s'y connecte et reçoit les indices de sa présence. Cette première connexion, avant sa réception par autrui, tient de l'apparition primaire, de ce que nous nommerons la «protophanie ». L'ontophanie ne se réalise que lorsqu'autrui perçoit le sujet. Un au-delà de l'ontophanie existe également. Dès lors qu'un message est reçu, qu'un contact est ajouté, son profil apparaît sur mon espace personnel qu'il soit actuellement présent sur la plateforme ou non - son statut est d'ailleurs indicé par la plateforme (" connecté », « déconnecté », « ne pas déranger », etc.). Le sujet existe alors en ligne à travers moi, dans mon espace, par ma connexion et notre relation. Cette forme d'apparition, nous la nommons « 
transphanie ». La protophanie est à la subjectivité ce que l'ontophanie est à l'intersubjectivité et la transphanie à la transsubjectivité.

\section{Conclusion}

Ainsi l'identité en interaction numérique fait l'objet d'un processus de construction complexe et notre approche phénoménolinguistique est motivée par la volonté d'analyser son émergence interactionnelle en prenant en compte ses modalités phénoménologiques d'énaction. Bien qu'étant désormais deux disciplines distinctes, analyse interactionnelle et phénoménologie reste d'une part épistémologiquement liées et d'autre part se rejoignent dans une compréhension de l'identité comme phénomène intersubjectif. L'analyse phénoménolinguistique favorise alors la mise en lumière des étapes du processus intersubjectif de construction des identités. Elle permet en outre de percevoir l'évolution diachronique des identités et relations interindividuelles. Il s'agit d'appréhender l'identité comme phénomène interactionnel à partir de l'expérience qu'en font les sujets. Ainsi l'approche pluridisciplinaire nous permet d'apprivoiser tant la complexité de la notion d'identité que la complexité de ses modalités d'apparition.

\section{Bibliographie}

1. L. Greco, L. Mondada, Les identités-en-interaction : vers une approche multidimensionnelle, in L. Greco, L. Mondada, P. Renaud (dir.) Identités en interaction, Lambert Lucas (2014)

2. J. Denouël, Les interactions médiatisées en messagerie instantanée, organisation située des ressources sociotechniques pour une coprésence à distance. Thèse en sciences du langage: Université Paul Valéry Montpellier III (2008)

3. M. Merleau-Ponty, Phénoménologie de la perception. Paris : Gallimard (1945)

4. C. Kerbrat-Orecchioni, Le discours en interaction, Paris : Armand Colin (2005)

5. E-M. Lipiantsky, L'identité dans la communication, in Communication et langages 97, p. 31-37 (1993).

6. E. Goffman, La mise en scène de la vie quotidienne, 1. La présentation de soi. Paris: Les éditions de minuit (1973)

7. M. Neyraut, Alter Ego, Paris : Édition de l'Olivier, coll. «Penser/Rêver » (2008)

8. F. Georges, Représentation de soi et identité numérique, in Réseaux 154 (2009)

9. A. Casilli, «Être présent en ligne » in Idées économiques et sociales 169, p.16-29. (2012)

10. M. Heidegger, Etre et temps, Paris : Gallimard (1972)

11. J-P. Sartre, L'être et le néant, Paris : Gallimard (1943)

12. S. Vial, L'être et l'écran, Paris : Presses Universitaires de France (2013)

13. P. Lévy, Qu'est-ce que le virtuel ?, Paris : La découverte (1998)

14. M. Serres, Atlas. Paris : Julliard (1994) 